\title{
A Proposed Architecture for Finding Missing Threads in Internet
}

\section{Saravanan}

Faculty of Operations \& ITICFAI Business School (IBS), Hyderabad,The ICFAI Foundation for

Higher Education (IFHE)(Deemed to be university u/s 3 of the UGC Act 1956)Hyderabad-India

\section{ABSTRACT}

As increasing the usage of internet grows very rapidly present days. It creates challenge for my researchers how the connectivity links works such a heterogeneity network. Particularly on individual domain how the procedure works to transfer data or communication among the network. Identifying the structure at the individual level still one of the most demanded research activity. Here most of the researchers don't know where the missing procedure or how the missing procedure change our network structure. Identify the missing procedure it is necessary to find the network architecture completely. In this paper a new architecture helps to find the missing thread on the internet.

KEY WORDS: NETWORK ARCHITECTURE, MISSING CONNECTION, NETWORK PROCEDURE, INDEPENDENT SYSTEM, ROUTING PROCEDURE, PERFORMANCE EVALUATION.

\section{INTRODUCTION}

It is highly necessary for finding the network structure whenever user find difficult in mission connection. This work highly complicated in individual network structure (Floyd \& Paxson 2001; Chang et al. 2004). For that it is necessary user need to build correct and absolute structure for network operations. There are various methods are currently existing for finding missing connection. One important observations identified most of this missing connection take place in peer-top-peer type of network structure only (Colitti et al. 2007; Cohen \&t Raz 2006). This take place especially at network exchange point or network exchange node. But constructing the accurate and obsolete network design is one of challenging factor for many researchers. In most of the existing systems collects the network steering information's that are all recorded in a table (Saravanan 2016). This table entry are updates at

\section{ARTICLE INFORMATION}

*Corresponding Author: sa_roin@yahoo.com

Received 11th Oct 2020 Accepted after revision 30th Dec 2020

Print ISSN: 0974-6455 Online ISSN: 2321-4007 CODEN: BBRCBA

Thomson Reuters ISI Web of Science Clarivate Analytics USA and Crossref Indexed Journal

\section{Clarivate
Analytics}

NAAS Journal Score 2020 (4.31)

A Society of Science and Nature Publication,

Bhopal India 2020. All rights reserved.

Online Contents Available at: http//www.bbrc.in/

Doi: http://dx.doi.org/10.21786/bbrc/13.13/56 regular interval or whenever any transaction take place on the network structure. Additionally, network steering registries helps the user find the missing connection (Xu et al. 2004). Using this resources user identify the missing connection. There is no guarantee that each of this resources are providing complete details to the user, sometime providing information may be incorrect or inaccurate also. So user first need to find the gene unity of the available datasets. In most of research works network swapping points are not yet consider or this points are not included in network architecture (Lakhina 2003). This points normally consider least significant points. For that most of the research work devoted first find the missing connection, find the importance of the missing connection. Based on this information user need to find the missing connections.

\section{Existing System}

- Most of the missing connection details are identified through network swapping points.

- Information's that are all recorded and collected though network steering table

- Missing connection are identified based on the network architecture. For that it is necessary precise and outmoded structure (Oliveira et al. 2007)

\section{Proposed System}

- Initially work starts with missing links also identifies

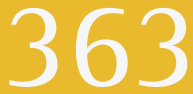


this link change any network stricture.

- $\quad$ Entire work divided into two steps process. First find the missing link, find the network structure.

- Find network swapping points. This helps to find more exchange point in our network. This process shown in the fig 1 .

Figure 1.1: Proposed System

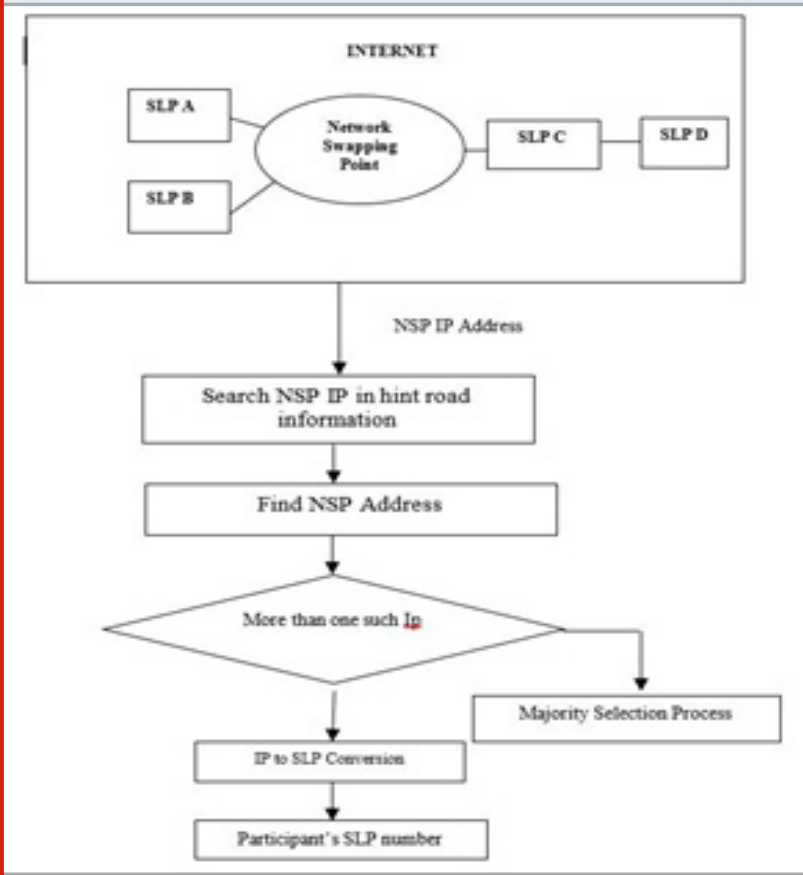

Experimental Setup:

Patterns of the Peer-Peer Edges: Normally network systems transfer information from one device to other without user's knowledge. Especially in End-to-End transmission any communication or any information's are transferred from one device to other in terms of message or communication through the addressing concepts (Mao 2003). This can be done either direct or indirect transmission mechanism. This transmission done with help of end-to -end communication network or end -to-end server. This communication are controlled and monitored with help of steering board (Saravanan 2016). This board identifies all the devices connected in the network it will select any one of the device as the first receiver then start transmit the message or communication. This first receiver after receiving the information or communications it starts transmit the same to the rest of network devices. This model is shown in the fig 1.2 .

Figure 1.2: Patterns of The End-to-End Edges

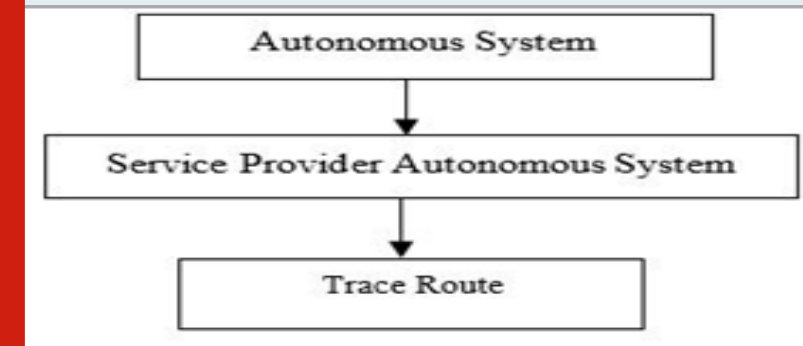

Patterns of the Service-Provider Edges: In End-toEnd communication network single device selected as a first receiver through this rest of the devices in the network receives information or communications. Along this an examination supplier helps to provide rest of the services in the network (Mao et al. 2003; Mallick et al. 2019; Mallick et al. 2020). Normally these setups act as a resource provider for many business and commercial applications. It is shown in the fig 1.3. They provide various resources such as storage resource, communication resource or processing certain operational resource or the combination of these three services.

Figure 1.3: Patterns of The Service-Provider Edges

Service Provider Autonomous System

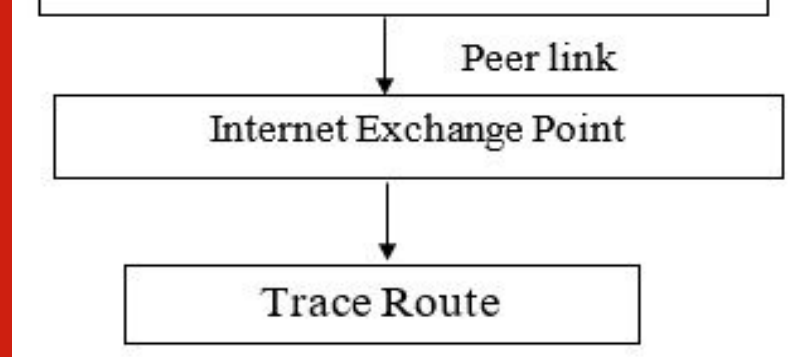

Discovering Broken Threads in Internet: This procedure explains the missing thread on the internet. It works based on the new created self-routing procedure. With help of self-routing procedure track the user's information effectively. For that first user need to collect the self-routing procedure and the correspond IPad dress. This way user can easily have identified the missing information. This process shown in the fig 1.4.

Figure 1.4: Discovering Broken Threads in Internet

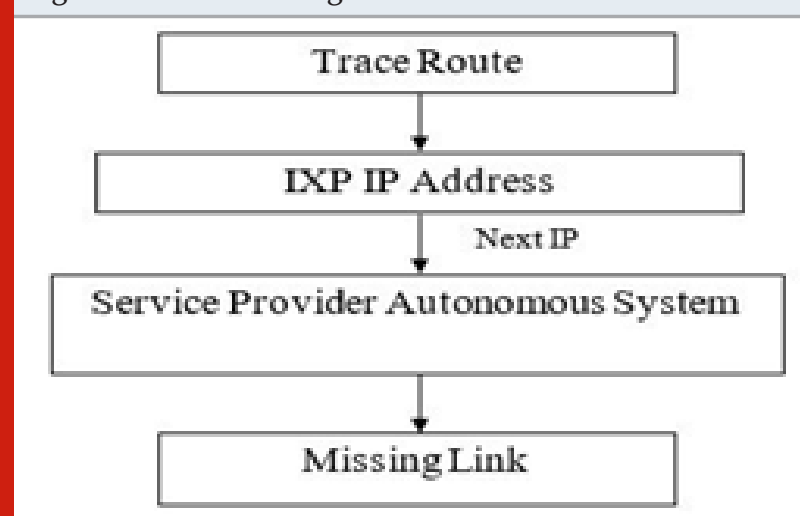

Experimental outcomes: The proposed system works with missing link and identifies the network structure. Discovering of peer- peer edge, service provide edges and broken threads are constructed and tested with various inputs. The result of proposed work is shown in the fig 1.5-1.19. 
Figure 1.5: Trace route in Machine 1

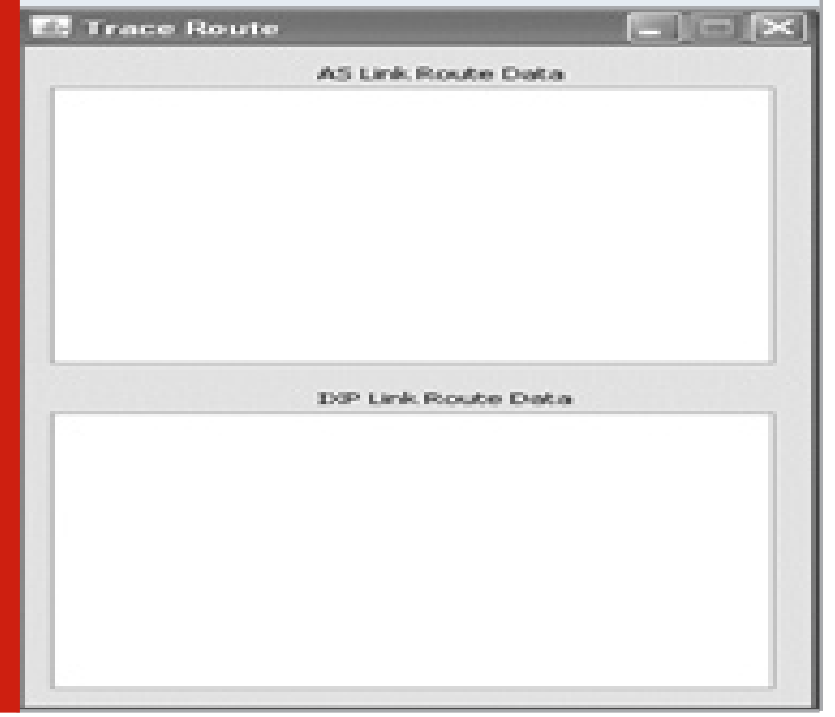

\section{Message}

\section{1) Enter DP IPAddress \\ 165.1.1.0}

$$
\text { OK }
$$

\section{Cancel}

Figure 1.8: SPAS in Machine 2

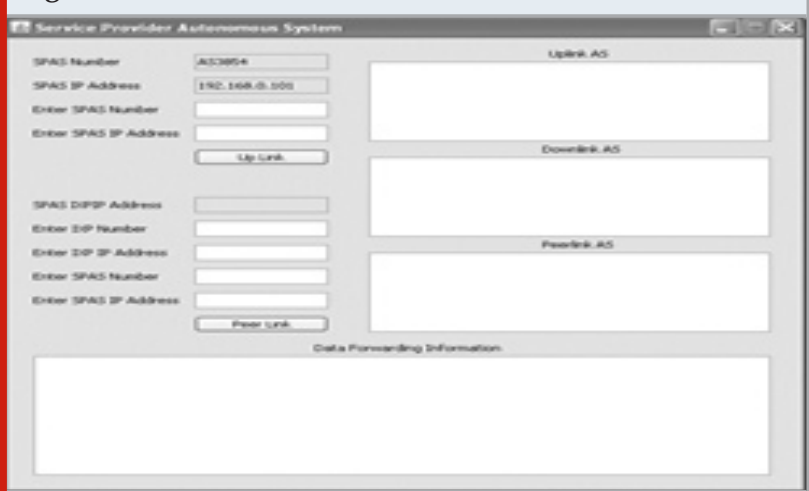

Figure 1.9: AS in Machine 3

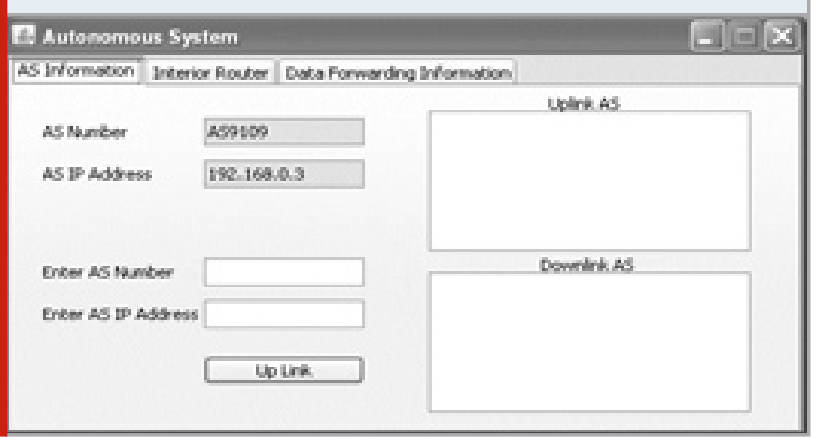

Figure 1.6: IXP in Machine 1

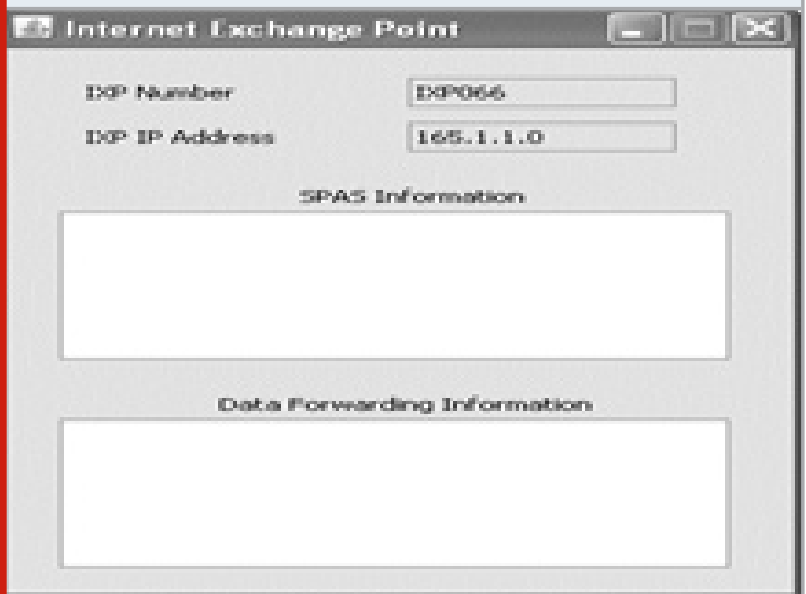

Figure 1.7: SPAS in Machine1



Figure 1.10: Host in Machine 3

\section{Message}

1. Enter Host IPAddress

20.301.1.1

OK Cancel

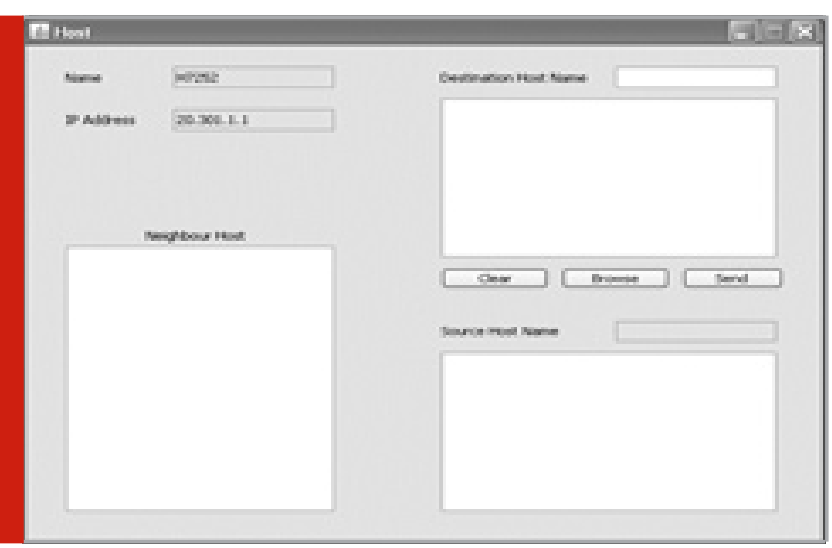


Figure 1.11: Machine 3 AS Shows All the Host and Router IP Address

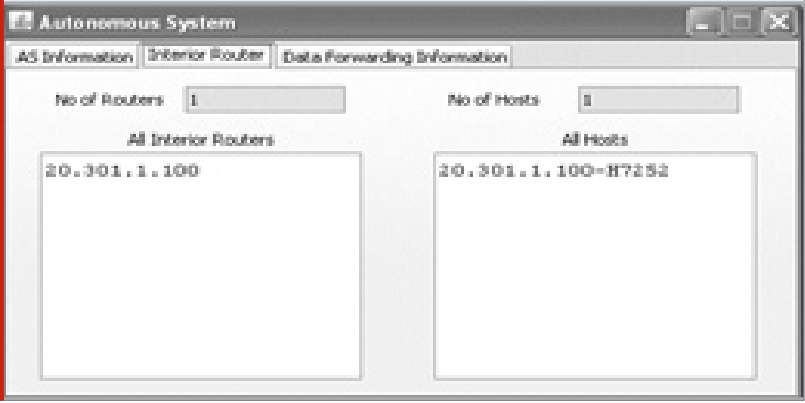

Figure 1.12: AS in Machine 4

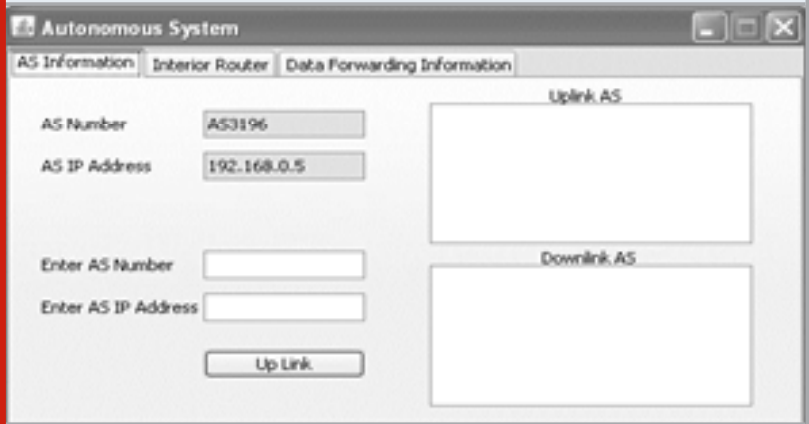

Figure 1.14: Machine 3 AS is uplinked with SPAS in Machine 1

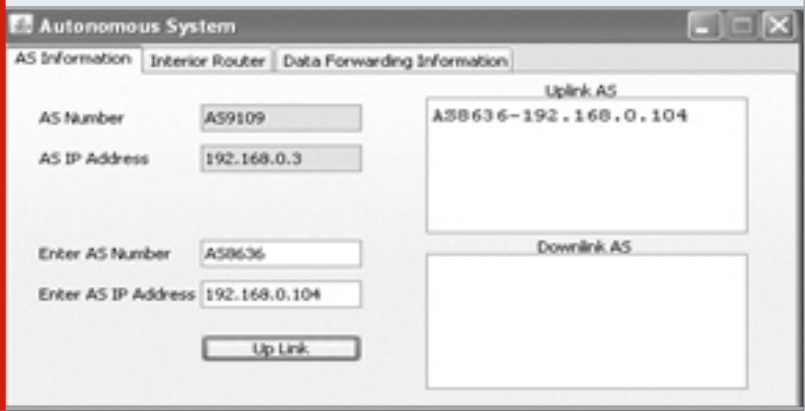

Figure 1.16: Machine 3 AS is uplinked with Machine 2 AS

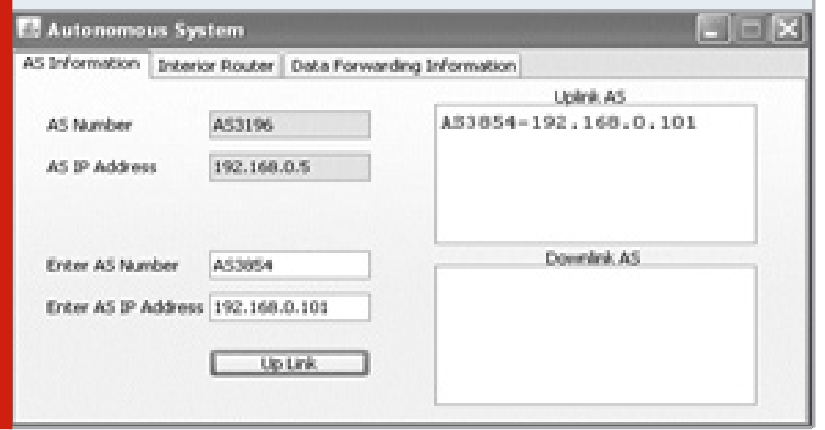

Figure 1.13: Host in Machine 4 and Machine 4 AS shows all the Host and Router IP address
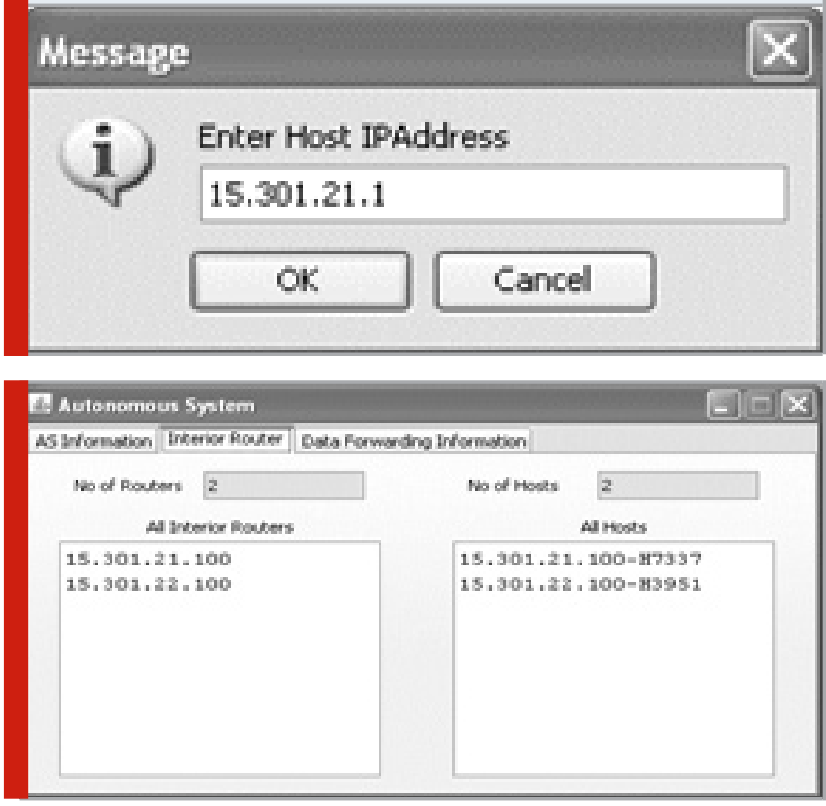

Figure 1.15: Machine 1 SPAS shows the downlink

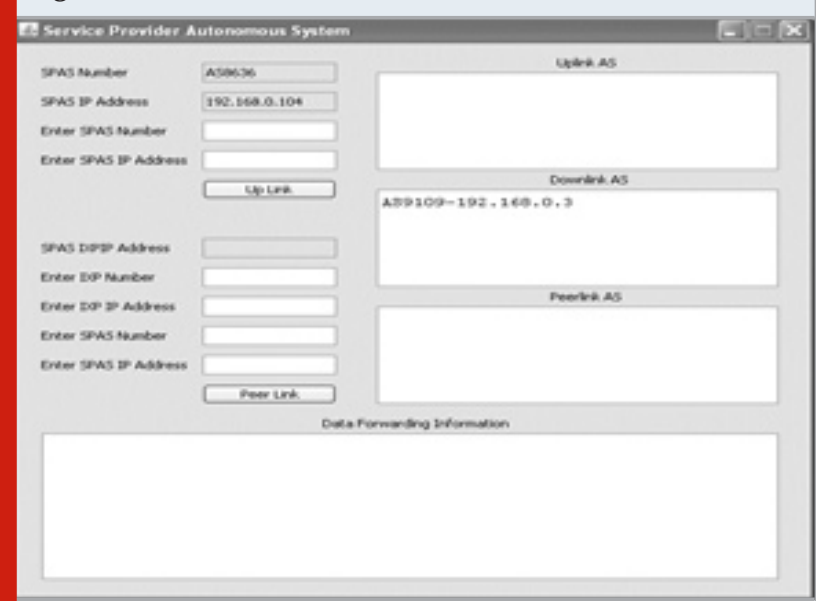

Figure 1.17: Machine 2 SPAS shows the downlink AS

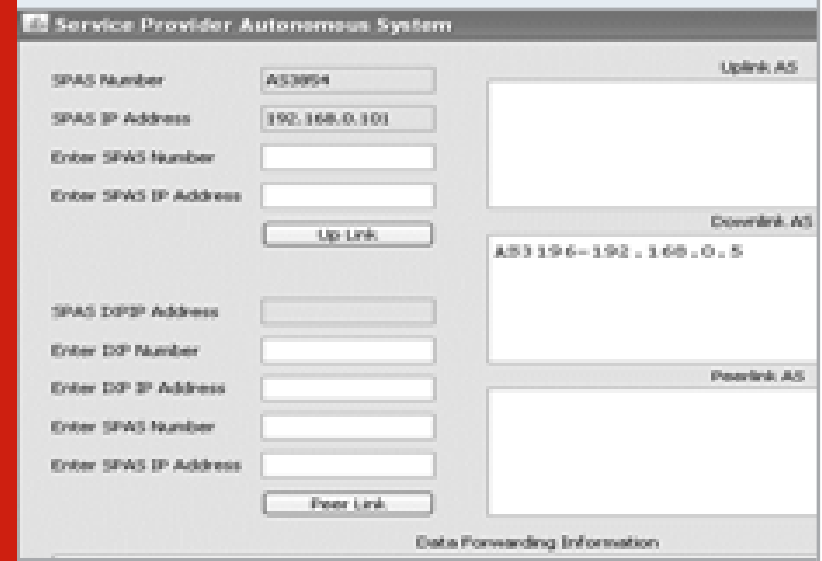


Figure 1.18: Trace Route in Machine 1 shows all the IP address

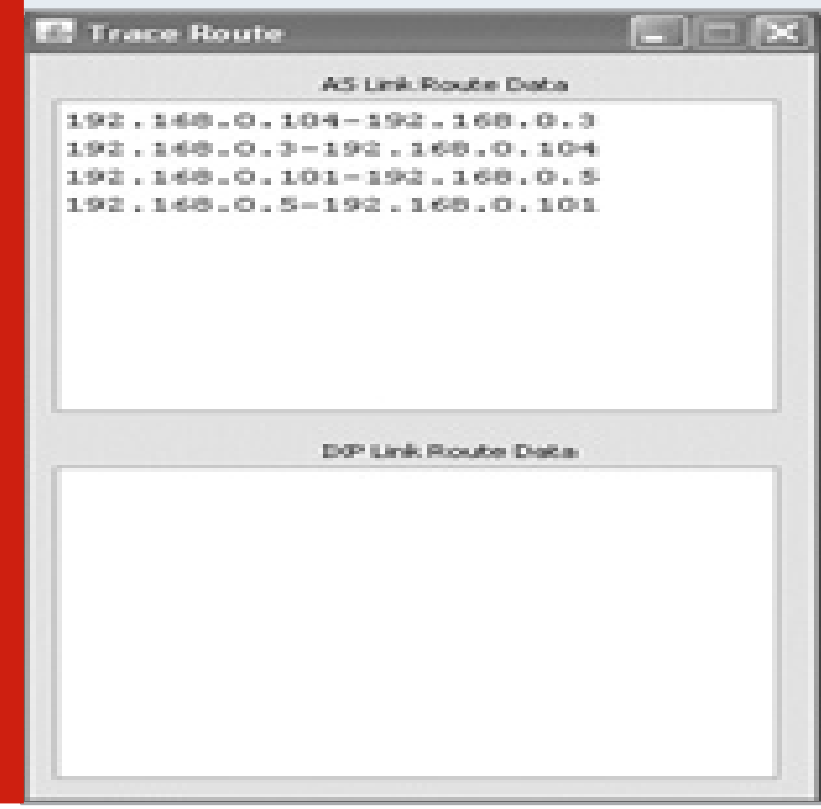

\section{CONCLUSION}

Experimental setup identifies easily how many missing connecting in the network architecture through help of steering counter. Experimental outcomes also identify that IXPs are identified with help of end-to-end AS link. From the outcomes user observed that very few IXPs are circulate this information on steering counter. Information's available on the steering counter are not accurate and most reliable one.

\section{REFERENCES}

A. Lakhina, J. W. Byers, M. Crovella, and P. Xie, (2003) Sampling biases in IP topology measurements," in Proc. IEEE INFOCOM, vol. 1, pp. 332-341.

D. Achlioptas, A. Clauset, D. Kempe, and C. Moore, (May 2005) On the bias of trace route sampling, or powerlaw degree distributions in regular graphs," in Proc. ST0C'05, Baltimore, MD, pp. 694-703.

D.Saravanan, (2016) Design and implementation of feature matching procedure for video frame retrieval", International journal of control theory and applications, 9(7), Pages 3283-3293.

D.Saravanan" Segment Based Indexing Technique for Data file “, Procedia of computer Science,87(2016), Pages 12-17, ISSN: 1877-0509, June 2016

H. Chang, R. Govindan, S. Jamin, S. Shenker, and W. Willinger, "Towards capturing representative AS-level Internet topologies," Computer Networks, vol. 44, no. 6, pp. 737-755, 2004.

K. Xu, Z. Duan, Z. Zhang, and J. Chandrashekar, “On properties of Internet exchange points and their impact
Figure 1.19: Machine 1 Trace Route shows all the AS link route data and IXP link route data

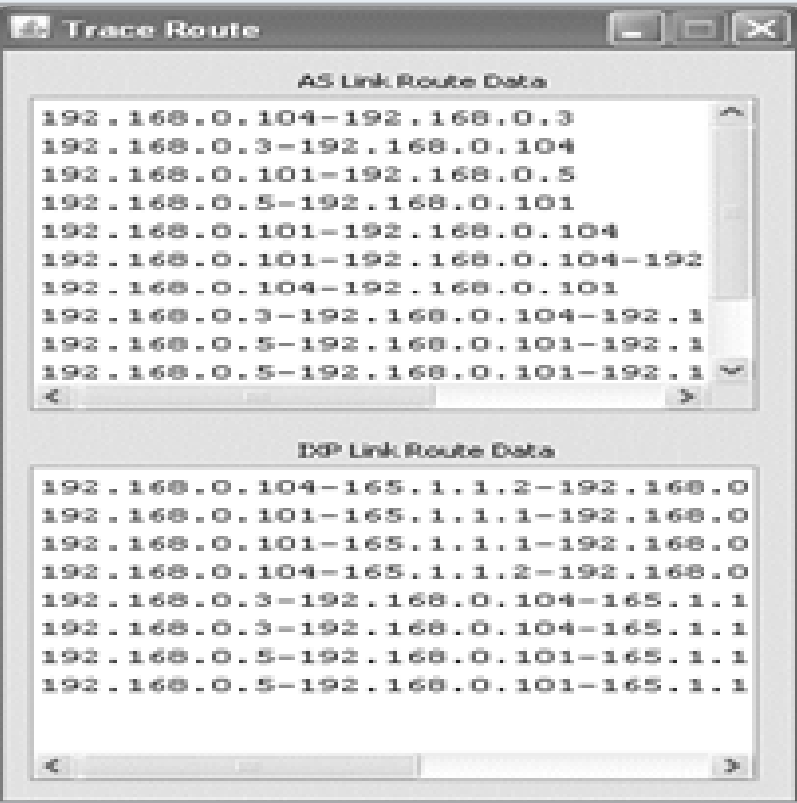

on AS tolology and relationship," in Networking, 2004, pp. 284-295.

L. Colitti, G. DiBattista, M. Patrignani, M. Pizzonia, and M. Rimondini, "Investigating prefix propagation through active BGP probing,” Microprocessors Microsyst., vol. 31, no. 7, pp. 460-474, 2007.

Mallick P. K., Balas V. E., Bhoi A. K., and Chae G.-S. (Eds.): Cognitive Informatics and Soft Computing: Proceeding of CISC 2019, Vol. 768, (2020).

Mallick P. K., Balas V. E., Bhoi A. K., and Zobaa A. F. (Eds.): Cognitive Informatics and Soft Computing: Proceeding of CISC 2017, Vol. 768, (2019).

R. Cohen and D. Raz, "The Internet dark matter-on the missing links in the AS connectivity map," in Proc. IEEE INFOCOM, 2006, $12 \mathrm{pp}$.

R. V. Oliveira, B. Zhang, and L. Zhang, "Observing the evolution of Internet AS topology," in Proc. ACM SIGCOMM, 2007, pp. 313-324.

S. Floyd and V. Paxson, "Difficulties in simulating the Internet," IEEE/ACM Trans. Networking, vol. 9, no. 4, pp. 392-403, Aug. 2001.

Saravanan," Image frame mining Using indexing technique" Data Engineering and Intelligent Computing, SPRINGER Book series, Chapter 12, Pages 127-137, ISBN:978 -981-10-3223-3, July 2017

Z. M. Mao, D. Johnson, J. Rexford, J. Wang, and R. Katz, "Scalable and accurate identification of AS-level forwarding paths," in Proc. IEEE INFOCOM, 2004, vol. 3, pp. 1605-1615.

Z. Mao, J. Rexford, J. Wang, and R. Katz, "Towards an accurate AS-level trace route tool," in Proc. ACM SIGCOMM, 2003, pp. 365-378. 